\title{
1 Das Phänomen der Gleichzeitigkeit in Interaktionen
}

Die Gleichzeitigkeit kommunikativer Elemente ist ein Phänomen, das in unterschiedlicher Ausprägung in vielen Bereichen menschlicher Face-to-Face-Interaktion beobachtet werden kann. Dazu zählen unter anderem die sequentielle Gleichzeitigkeit, die multimodale Simultanität und die gleichzeitige Relevanz multipler Aktivitäten. Ein typisches Beispiel für sequentielle Gleichzeitigkeit ist das zeitgleiche Sprechen mehrerer Personen. Multimodale Simultanität bezeichnet die Möglichkeit von Menschen die Ressourcen ihrer Körper so einzusetzen, dass sie zum Beispiel zeitgleich sprechen und gestikulieren können. Diese multimodale Organisation von Interaktion ist die Voraussetzung für die dritte Form der Gleichzeitigkeit, die gleichzeitige Relevanz multipler Aktivitäten. Dieses in der vorliegenden Studie fokussierte Phänomen zeigt sich in interaktionalen Kontexten dann, wenn Menschen ihre Ressourcen so einsetzen, dass sie damit mehrere Aktivitäten gleichzeitig realisieren können. $\mathrm{Zu}$ beobachten ist dieses Phänomen zum Beispiel in solchen Situationen, in denen Menschen im Verlauf eines gemeinsamen Kochens Speisen zubereiten und simultan anderen Beteiligten Anweisungen bezüglich der nächsten Arbeitsschritte geben.

Während die ersten beiden Formen der Gleichzeitigkeit in der Interaktionsforschung bereits einschlägig beschrieben worden sind (vgl. 2.2), ist das als multiactivity (Haddington et al. 2014) konzeptualisierte Phänomen des simultanen Vollzugs mehrerer Aktivitäten kaum bearbeitet worden (zum aktuellen multiactivity-Forschungsstand vgl. 2.1). Dies hat mehrere Gründe. Zum Ersten ist der größte Teil der aktuellen ethnomethodologisch-konversationsanalytischen Studien trotz „modalitätsspezifischer Erweiterung“ (Schmitt 2015: 44) nach wie vor vornehmlich an der Rekonstruktion verbaler Strukturen interessiert, wodurch die gleichzeitige Relevanz sprachlicher und besonders nichtsprachlicher Vorgänge aus dem Forschungsinteresse rückt. ${ }^{1}$ Zum Zweiten herrscht im Rahmen interaktionsanalytischer Arbeiten bislang keine Einigkeit darüber, wie Aktivität als Konzept überhaupt zu fassen wäre, was die Betrachtung gleichzeitiger Relevanz mehrerer Aktivitäten deutlich erschwert (zum unterschiedlichen Gebrauch des Aktivitätskonzepts in der Interaktionsforschung vgl. 2.3). Dieser Studie liegt eine Auffassung von Aktivitäten zugrunde, die eng an interaktionale Vorgänge der Teilnahme an Interaktionen (participation, Goodwin \& Goodwin 2004; Schmitt

1 Beispiele für Ausnahmen von dieser Tendenz bilden Studien zu Interaktionen beim Tanzen (Keevallik 2015) oder zur intersubjektiven Herstellung einer Verkostung in Mondada (2018b). 
2012; Goffman 1979) gebunden ist. Aktivitäten werden in dieser Arbeit demnach als interaktionale Einheiten verstanden, die durch die (auch verbal abstinenten, vgl. Heidtmann \& Föh 2007) Beteiligungsweisen der Interagierenden hervorgebracht werden (vgl. 2.3.1). In diesem Sinne wird Multiactivity nicht notwendigerweise als Phänomen gleichzeitigen Handelns, sondern primär als koordinativer Prozess der Partizipation an mehreren Aktivitäten betrachtet. Zum Dritten variieren in den aktuellen Studien die Zeitspannen zwischen zwei Ereignissen, die als zeitgleich ablaufend gefasst werden. Während Mondada (2014c: 71-72) beispielsweise beobachtet, dass scheinbar gleichzeitige Vorgänge bei genauerer Betrachtung gar nicht simultan, sondern als „dynamic alternations of successive micro-actions" vollzogen werden, zeigt Hoey (2018a) die Praktiken, mit denen Interagierende selbst strukturell inkompatible Aktivitäten wie TRINKEN ${ }^{2}$ und BERICHTEN zur selben Zeit realisieren. Ein Grund für diese Diskrepanz in der Beschreibung von Gleichzeitigkeit könnte das zugrundeliegende Verständnis von Zeit (als temporalem Punkt) bzw. Dauer (Abstand zwischen zwei Zeitpunkten) sein, was eine knappe Bestimmung des Zeit-Begriffs notwendig werden lässt, der für diese Studie gelten soll.

Zeit kann im Newton'schen Sinneals etwas Absolutes und Messbares (Hawking 1988: 18) und somit als äußere Zeit verstanden, oder im Sinne von Bergson (2016 [1920]) als innere Dauer gefasst werden. Diesem Verständnis von Dauer als „eine[r] durch das Bewußtsein hergestellte[n] Einheit einzelner Erlebnisinhalte“ (Nassehi 2008: 59) folgt Husserl $(2013,1928)$ in seiner Phänomenologie. Er entwickelt dieses Verständnis vom Erleben eines inneren Zeitbewusstseins weiter und blendet dazu die objektive Zeit vollständig aus. Auch bei Schütz \& Luckmann (2017 [1979]) findet sich in der Beschreibung der zeitlichen Struktur der Lebenswelt die subjektive Zeit des Bewusstseinsstroms, die sich hier jedoch - im Unterschied zu Bergson und Husserl - mit einer „Rhythmik des Körpers“ (Schütz \& Luckmann 2017 [1979]: 84) als biologischer und der „Welt-Zeit“ (ebd.: 81f.) als sozialer Zeit überschneidet. Allen drei Ansätzen ist unter anderem gemein, dass sie davon ausgehen, dass Menschen die innere Zeitwahrnehmung subjektiv erleben. Dies kann zum einen durch Erkenntnisse aus der Psychologie ergänzt werden, die beispielsweise zeigen, dass die scheinbare Beschleunigung der Zeit mit zunehmendem Alter keine subjektiv gefühlte Alterserscheinung per se ist, sondern ebenso aus dem Umgang ${ }^{3}$ der jeweiligen Personen mit der Zeit und anderen Menschen erwachsen kann (Wittmann et al. 2015; Winkler et al. 2017). Zum anderen basiert die innere Zeit in interak-

2 Immer wenn im Folgenden eine Aktivität gemeint ist, wird in Abgrenzung zu verwandten Konzepten wie Praktiken oder Handlungen eine Formatierung in KAPITÄLCHEN verwendet.

3 Dazu zählen die Autor`innen unter anderem Routinen, Langeweile und positive wie negative Einstellungen bezüglich bestimmter Zeitabschnitte. 
tionalen Kontexten auch auf der geteilten Wahrnehmung aller Beteiligten einer Situation. Dies zeigt sich besonders in der zeitlich geordneten sequentiellen Organisation von Interaktionen, die der Garfinkel'schen (2006 [1948], 1967) Ethnomethodologie zugrunde liegt. Demnach verweisen Interagierende in ihren Handlungen auf ihr Verständnis einer laufenden Aktivität und teilen miteinander ihre Interpretation eines Interaktionsbeitrags als „public and shared dimension of time“ (Rawls 2005: 173). Für die Konzeption von Gleichzeitigkeit in Interaktion, die im Folgenden für diese Studie gelten soll, bedeutet das, dass gleichzeitig vollzogene Aktivitäten nicht zwangsläufig messbar (im Sinne einer äußeren Zeit) und zeitlich kongruent zueinander verlaufen müssen, sondern im Sinne einer inneren Zeit von den Beteiligten als gleichzeitig realisiert wahrgenommen werden. Diese Wahrnehmung von Gleichzeitigkeit, die sich öffentlich in den koordinativen Handlungen der Interagierenden ausdrückt, wird im weiteren Verlauf als gleichzeitige Relevanz multipler Aktivitäten bezeichnet.

Deutlich wird dies an folgendem Beispiel, das eine Situation kurz vor Beginn einer Theaterprobe zeigt (zur Beschreibung der im Rahmen dieser Studie verwendeten Daten und dem aktuellen Forschungsstand zu Theaterproben als Untersuchungsmaterial vgl. Kap. 4). In dieser gehen eine Regieassistentin (ASS) und eine Hospitantin (HOW) der gemeinsamen Aktivität (im Folgenden: Ensembleaktivität, vgl. 2.3.2) ABGLEICHEN voN WISSENSBESTÄNDEN nach, während die Regieassistentin im Rahmen einer individuellen Aktivität (im Folgenden: Einzelaktivität, vgl. ebd.) zur gleichen Zeit ISST und die Hospitantin ihren MANTEL ABLEGT. Die beiden Interagierenden zeigen einander ihr Verständnis der gleichzeitigen Relevanz der multiplen Aktivitäten dadurch an, dass sie ihre Einzelaktivitäten genau dann pausieren, als die Ensembleaktivität einen kritischen Punkt (eine Alternative wird angeboten) erreicht und wieder fortsetzen, als dieser überwunden ist. Im Vorfeld des folgenden Transkriptausschnitts bietet die Regieassistentin der Hospitantin eine Führung durch das Theater an. Die Hospitantin lehnt das Angebot dadurch ab, dass sie mitteilt einige Arbeitsbereiche des Theaterhauses bereits zu kennen. Sie sei ferner mit der Ausstatterin verabredet, die Hilfe mit den Kostümen benötige. An dieser Stelle setzt das untenstehende Transkript ein, das sich in drei Koordinationsphasen einteilen lässt. Zu Beginn a) GLEICHEN die beiden Teilnehmerinnen ihre WISSENSBESTÄNDE bezüglich wichtiger Orte im Theater miteinander Ав (Requisite) und handeln im Zuge dessen aus, ob und wie sie die anschließende Theaterführung stattfinden lassen - während sie gleichzeitig ESSEN bzw. den MANTEL ABLEGEN. Im Anschluss daran b) pausieren beide Interagierende ihre Einzelaktivitäten EsSEN bzw. MANTEL ABLEGEN, um eine potentielle Alternative auszuhandeln. Als c) diese Alternative schließlich bearbeitet worden ist, setzen beide Interagierende ihre pausierten Einzelaktivitäten ESSEN bZW. MANTEL ABLEGEN fort. 


\section{Transkript A: Die gleichzeitige Relevanz der Aktivitäten WISSENSBESTÄNDE ABgLeichen Und MANTEL ABLEGEN bZW. ESSEN ${ }^{4}$}

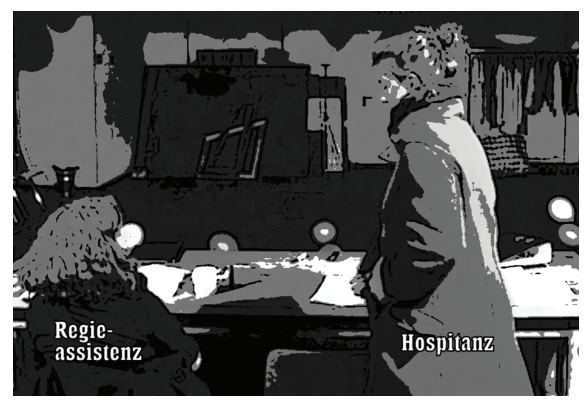

Abb. A.1: HOW öffnet ihre Mantelknöpfe.

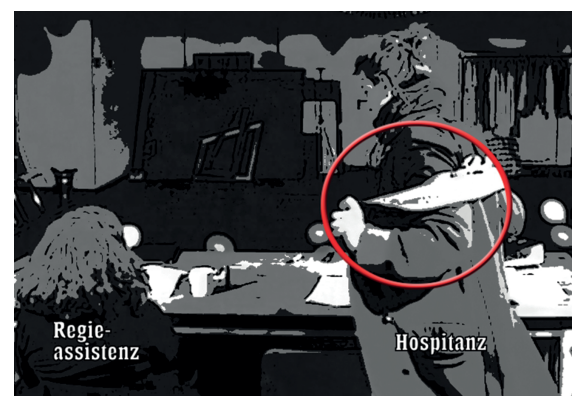

Abb. A.2: Der Mantel bleibt auf „halber Höhe“ (Kreis).

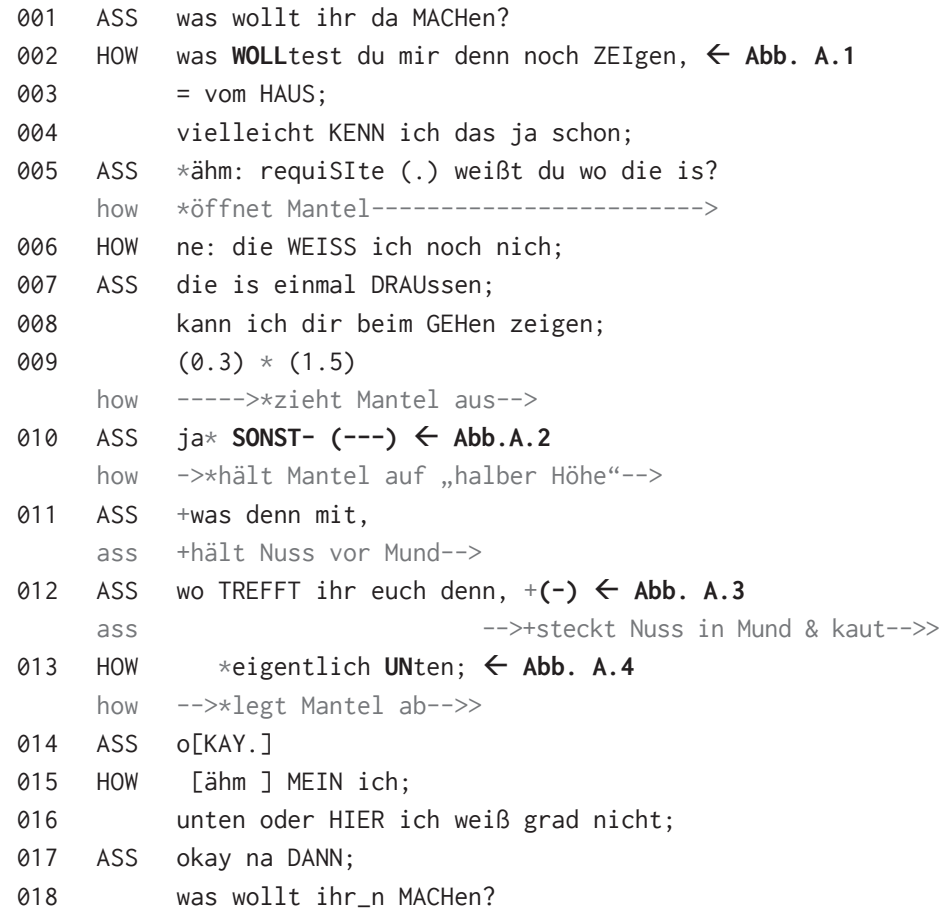

4 Die verwendete Transkription basiert auf dem Gesprächsanalytischen Transkriptionssystem GAT 2 (Selting et al. 2009). Die multimodale Annotation folgt Mondada (2018a, 2019b). Eine Übersicht der verwendeten Notationskonventionen findet sich im Anhang. 


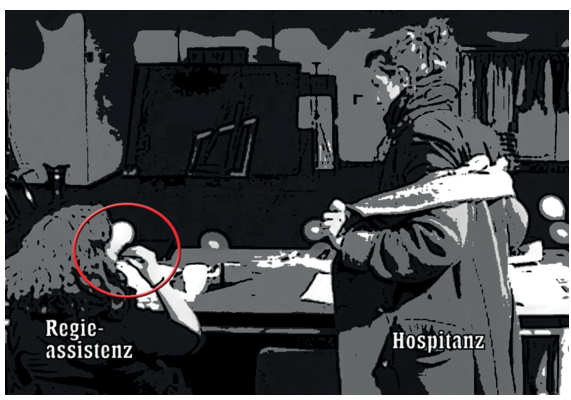

Abb. A.3: ASS isst eine Nuss (Kreis).

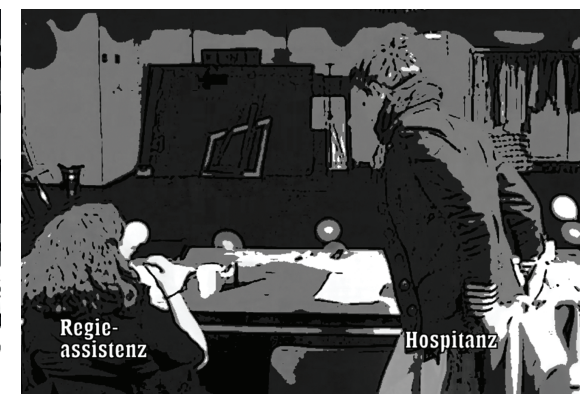

Abb. A.4: HOW legt den Mantel ab.

a) Gemeinsames ABGLEICHEN DER WISSENSBESTÄNDE bei simultanem Vollzug der Einzelaktivitäten (Z.001-009): Die Regieassistentin reagiert auf die Ablehnung ihres Angebots zu einer Theaterführung mit einer Frage an die Hospitantin nach ihrem Plan mit der Ausstatterin (Z.001). Diese begegnet der Frage, indem sie sich bei der Regieassistentin nach den Stationen der Führung erkundigt (Z.002). Sie liefert den Account für diese Gegenfrage nach, indem sie auf ihr potentielles Theaterwissen verweist (Z.004), das eine Führung unnötig werden lassen und den Konflikt beider möglicher Folgeaktivitäten (THEATERFÜHRUNG MIT DER REGIEASSISTENTIN vs. VERABREDUNG MIT DER AUSSTATTERIN) auflösen könnte. Während die Regieassistentin daraufhin überprüft, welches Wissen tatsächlich vorliegt (Z.005), beginnt die Hospitantin ihre Mantelknöpfe zu öffnen (Abb. A.1). Sie vollzieht damit ihre Einzelaktivität MANTEL ABLEGEN. Die Hospitantin bearbeitet diese Aktivität mit haptischen Beteiligungsweisen, während sie gleichzeitig mit Blickkontakt, Körperorientierung und verbalen Beiträgen (Z.006) an der Ensembleaktivität mit der Regieassistentin teilnimmt. Auf diese Weise realisiert die Hospitantin simultan zwei Aktivitäten, ohne dass sie oder die Regieassistentin in der Gestaltung der Ensembleaktivität bzw. in ihrem sonstigen Verhalten anzeigen würden, dass diese Simultankoordinierung der Aktivitäten im Konflikt mit einer anderen Handlung stehen würde. Beiden Aktivitäten wird somit durch die Teilnehmenden zunächst derselbe Stellenwert innerhalb der laufenden Situation zugeschrieben.

\section{b) Pausieren bzw. Verzögern der Einzelaktivitäten MANTEL ABLEGEN und ESSEN} zugunsten der Ensembleaktivität (Z.010-012): Der Vollzug der Aktivitäten verändert sich, als die Regieassistentin nach einem zuerst angebotenen Kompromiss, sie könne der Hospitantin die Requisite „beim GEHen zeigen“ (Z.008), eine Alternative andeutet (,ja SONST“, Z.010). Zeitgleich mit dieser Äußerung der Regieassistentin als Anakoluth hält die Hospitantin ihren Mantel „auf halber Höhe“ (Abb. A.2) fest und pausiert damit das MANTEL ABLEgen. Sie zeigt so an, dass sich für sie nun die Prioritäten innerhalb der gleichzeitig relevanten Aktivitäten 
hin zur Ensembleaktivität verschoben haben. Auch bei der Regieassistentin ist eine solche Priorisierung der Ensembleaktivität zu beobachten, als sie eine Nuss im Rahmen ihrer Einzelaktivität EsSEN zwar zum Gesicht führt, sie diese jedoch erst in den Mund steckt (Abb. A.3), als sie die projizierte Alternative einlöst und ihren Turn (Z.010-012) auf diese Weise als abgeschlossen markiert. Diese Interpretation zeigt sich in der Folgehandlung der Hospitantin, die daraufhin das Rederecht übernimmt (Z.013). Auffällig ist an dieser Stelle, dass das Pausieren und Fortsetzen der Einzelaktivitäten von den Beteiligten analog zur Projektion und Projektionseinlösung im Rahmen der Ensembleaktivität vollzogen wird. Das legt die Vermutung nahe, dass die Koordination der Aktivitäten hier vor allem funktionalen Gesichtspunkten folgt und weniger Ausdruck einer kognitiven Beanspruchung (cognitive load, vgl. Sweller 2010; Wickens 2008) ist. Die von beiden Interagierenden geteilte Wahrnehmung der Gleichzeitigkeit der Aktivitäten drückt sich also an dieser Stelle in der Bearbeitung der simultanen Relevanz der multiplen Aktivitäten aus. Indem beide Interagierende ihre Einzelaktivitäten pausieren bzw. zurückhalten, zeigen sie einander den erhöhten Stellenwert an, dem sie der Ensembleaktivität lokal zuschreiben.

c) Fortsetzung des simultanen Vollzugs der Aktivitäten (Z.012-018): Die Priorisierung der Ensembleaktivität löst sich wieder auf, als die Regieassistentin nach dem Treffpunkt der Hospitantin mit der Ausstatterin fragt (Z.012), womit sie möglicherwiese eruiert, inwiefern beide Folgeaktivitäten miteinander verbunden werden könnten. Indem die Regieassistentin zeitgleich ihre zurückgehaltene Einzelaktivität ESSEN realisiert, zeigt sie der Hospitantin ihre veränderte Interpretation der gleichzeitigen Relevanz der Aktivitäten innerhalb der Situation an. Die Hospitantin folgt dieser Interpretation. Sie nimmt nun ihrerseits ihre pausierte Einzelaktivität wieder auf (Z.013) und LEGT ihren MANTEL AB (Abb. A.4). Dadurch beteiligen sich beide Teilnehmerinnen an der Ensembleaktivität (Z.014-018), während sie ihre Einzelaktivitäten wieder simultan dazu vollziehen.

Wie die Rekonstruktion des obenstehenden Transkriptausschnitts zeigt, können die im Rahmen einer Aktivität vollzogenen Beteiligungsweisen die Teilnahme an einer anderen, gleichzeitig relevanten Aktivität beeinflussen. So verhindert die haptische Beteiligungsweise am KLEIDUNG ABLEGEN zwar eine gestische Beteiligungsweise an der Ensembleaktivität durch die Hospitantin, hält sie aber nicht davon ab, sich trotzdem simultan an beiden Aktivitäten zu beteiligen: Sie hält den Mantel auf „halber Höhe“, während sie gleichzeitig per Körperorientierung, Blickkontakt und verbalen Beiträgen am WISSENSBESTAND ABGLEICHEN partizipiert. Möglich wird dies dadurch, dass die Hospitantin ihre multimodalen Ressourcen so auf die Beteiligungsweisen der ko-relevanten Aktivitäten verteilen 
kann, ohne dass es zu einem Ressourcenkonflikt kommt. Die Beteiligungsweisen der für die Hospitantin gleichzeitig relevanten Aktivitäten verhalten sich somit strukturell kompatibel zueinander. Das bedeutet, dass keine der ko-relevanten Aktivitäten im Kern auf eine Beteiligungsweise zurückgreift, die auch für den Vollzug einer anderen Aktivität benötigt wird. Bei ihrer Interaktionspartnerin, der Regieassistentin, zeigt sich das gegensätzliche Phänomen der strukturellen Inkompatibilität von Beteiligungsweisen bei gleichzeitig relevanten Aktivitäten. Die Regieassistentin begegnet der strukturellen Inkompatibilität der Ensembleaktivität WISSENSBESTÄNDE ABGLEICHEN und der Einzelaktivität ESSEN dadurch, dass sie ihre sprachfreie Ess-Einzelaktivität verzögert, bis sie den Mund nicht mehr für verbale Beteiligungsweisen an der Ensembleaktivität benötigt. Da sich das eigentliche Nuss-Kauen im Gegensatz zum Nuss-in-den-Mund-Stecken durchaus strukturell kompatibel zur verbalen Beteiligung an der Ensembleaktivität verhält, kann die Regieassistentin danach weitere verbale Interaktionsbeiträge realisieren und trotzdem gleichzeitig ESSEN (Z.012-018).

Diese strukturelle (In)Kompatibilität der Beteiligungsweisen von ko-relevanten Aktivitäten ist eine der zentralen Anforderungen, auf die Menschen bei der Koordination multipler Aktivitäten reagieren müssen. Was die Betrachtung gleichzeitiger Relevanz multipler Aktivitäten in Interaktionen aus interaktionsanalytischer Perspektive so interessant macht, ist also weniger, mit welchen Ressourcen Menschen Aktivitäten in Interaktionen zeitgleich realisieren, sondern vielmehr die Frage nach der Bearbeitung struktureller (In)Kompatibilität multipler Aktivitäten:

1. Wie gehen Interagierende in sozialen Situationen mit der Anforderung um, wenn gleichzeitig zwei (oder mehr) Aktivitäten relevant werden, die simultan dieselben multimodalen Beteiligungsweisen erfordern?

Dieser Frage, die von der aktuellen multiactivity-Forschung bislang aufgrund der vorrangig sprachzentrierten Perspektive noch nicht bearbeitet worden ist (vgl. Forschungsstand 2.1), wird insbesondere im Rahmen von Kapitel 5 anhand einer Kollektion von 17 Fällen nachgegangen. Die Analysen zeigen, dass die Interagierenden unterschiedliche Verfahren (serielle, quasi-simultane und simultane Koordinierung) je nach Grad der strukturellen Kompatibilität der für die korelevanten Aktivitäten verwendeten Beteiligungsweisen einsetzen, um die gleichzeitig relevanten Aktivitäten abzubrechen/wiederaufzunehmen, zu pausieren/ fortzusetzen oder gleichzeitig zu vollziehen. Damit kann Gleichzeitigkeit eine von den Beteiligten situativ geteilte Erfahrung sein, mit der sie einander ihre lokalen Prioritäten der Aktivitäten und der damit verbundenen Interpretation der laufenden Interaktion anzeigen. Die Analyse vom obenstehenden Transkriptausschnitt 
A hat bereits einen Hinweis auf eine Systematik geliefert, die sich im Laufe der Studie zu einer Tendenz verdichten wird: Je strukturell inkompatibler die Beteiligungsweisen an ko-relevanten Aktivitäten sind, desto eher stellen Interagierende lokal weniger relevante Aktivitäten zurück. Wie die insgesamt 42 Fälle dieser Studie zeigen werden, handelt es sich bei der strukturellen (in)Kompatibilität von Beteiligungsweise an multiplen Aktivitäten um eine vergleichbar robuste Erklärung für Aktivitätsabbrüche, die - im Gegensatz zur multitasking-Forschung (z. B. Fintor et al. 2018; Schuch \& Konrad 2017) - ohne kognitive Überlegungen auskommt. Wenn die Beteiligungsweisen an multiplen Aktivitäten strukturell kompatibel sind, können Interagierende sie tatsächlich simultan vollziehen. Der diametrale Fall, also ein simultaner Vollzug von multiplen Aktivitäten, die sich in Bezug auf ihre Beteiligungsweisen strukturell inkompatibel zueinander verhalten, wurde von der multiactivity-Forschung bislang weitestgehend ignoriert. Aus diesem Desiderat ergibt sich die zweite Fragestellung dieser Studie:

\section{Unter welchen Bedingungen ist ein simultaner Vollzug multipler Aktivitäten trotz} struktureller Inkompatibilität der multimodalen Beteiligungsweisen möglich?

Dazu wird in Kapitel 6 zunächst die simultane Koordination strukturell kompatibler multipler Ensembleaktivitäten im Rahmen einer Tanzerarbeitung analysiert. Im Analysefokus der 16 Fälle stehen die Verfahren der Transformierung und Synchronisierung, mit denen die Beteiligten komplexe Aktivitätsverbünde herstellen, aufrechterhalten und gemeinsam zu einem Abschluss bringen. Das ist insofern sinnvoll, als die dort herausgearbeiteten koordinativen Verfahren der Transformierung und Synchronisierung auch Fällen des simultanen Vollzugs strukturell inkompatibler Beteiligungsweisen ko-relevanter Aktivitäten beobachtet werden können, die in Kapitel 7 betrachtet werden. Die dort präsentierten 10 Fälle umfassen die simultane Bearbeitung von strukturell inkompatiblen Einzelaktivitäten bei einer Szenenerarbeitung. Da sich die strukturelle Inkompatibilität in diesen Daten besonders hinsichtlich der visuellen Beteiligungsweise zeigt, wird im 7. Kapitel von mobilem Eye-Tracking als analytischem Zugriff auf diese koordinative Anforderung Gebrauch gemacht. Damit ist es möglich die Verfahren der Routinisierung und Priorisierung zu beschreiben, die die Teilnehmenden dazu einsetzen, um das kurzzeitige Fehlen einiger Beteiligungsweisen zu kompensieren und so die gleichzeitig relevanten Aktivitäten trotz struktureller Inkompatibilität simultan zu koordinieren. Die Betrachtung von simultan koordinierten Ensembleaktivitäten in koordinativen Kontexten ist durch die multiactivity-Forschung bislang nur am Rande erfolgt; der Fokus auf simultan vollzogene multiple Einzelaktivitäten fehlt bislang völlig. 
Ziel der Arbeit ist es somit, sowohl eine Systematik der Koordinationsverfahren multipler Aktivitäten in Abhängigkeit zur strukturellen (In)Kompatibilität der verwendeten Beteiligungsweisen zu präsentieren als auch die kommunikative(n) Funktion(en) der simultanen Koordinierung gleichzeitig relevanter multipler Aktivitäten in interaktionalen Kontexten zu beschreiben und damit aktuelle Desiderate in der multiactivity-Forschung zu bearbeiten. 